\title{
Distributed Renewable Energy under the Guidance of Price Autonomous Operation Technology
}

\author{
Arslan Habib $^{1 *}$, Adeel Arshad ${ }^{2}$, Rafiq Khan ${ }^{3}$ \\ ${ }^{1}$ School of Automation, Northwestern Polytechnical University, Xi'an, China \\ ${ }^{2}$ Fluids \& Thermal Engineering (FLUTE) Research Group, University of Nottingham, UK \\ ${ }^{3}$ School of Electrical Engineering, Xi'an Jiaotong University, Xi'an, China \\ Email: *Arslan.2010@live.com, adeel.arshad@nottingham.ac.uk, rafiqkhan2011@yahoo.com
}

How to cite this paper: Habib, A., Arshad, A. and Khan, R. (2017) Distributed Renewable Energy under the Guidance of Price Autonomous Operation Technology. Smart Grid and Renewable Energy, 8, 305-324.

https://doi.org/10.4236/sgre.2017.810020

Received: August 16, 2017

Accepted: October 10, 2017

Published: October 13, 2017

Copyright $\odot 2017$ by authors and Scientific Research Publishing Inc. This work is licensed under the Creative Commons Attribution International License (CC BY 4.0).

http://creativecommons.org/licenses/by/4.0/

\begin{abstract}
Penetration of Distributed Renewable Energy in active distribution network has increased year by year, and the distributed characteristics of active distribution network has become increasingly prominent; it is difficult for traditional centralized energy scheduling to solve the coordination of random output of distributed energy and the communication pressure of a large number of distributed data. In this paper, we propose a Distributed Renewable Energy Coordination Strategy based on price guidance, through hierarchical multi-agent model. The coordination model of each agent is introduced in detail, regional target, price coordination response strategy and regional security constraints, using Agent's Distributed Autonomy and Global Collaboration to realize the Energy Balance of Active Distribution Network and promote the Storage of Distributed Renewable Energy; the coordination strategy focuses on the impact of price adjustment on energy storage and flexible load response capacity to improve the distributed renewable energy consumption. Finally, through the quantitative analysis of the comprehensive performance of the index, the evaluation results of the traditional sequential simulation method are compared, and the rationality and validity of the proposed method are verified.
\end{abstract}

\section{Keywords}

Evaluation Index, Multi Agent, Distributed Renewable Energy, Price Coordination

\section{Introduction}

In the recent years, the distributed generation (DG) has been increased in active 
distribution network and this is because of rapid development of intermittent renewable energy generation represented by wind and photovoltaics. Active component types and quantities have been increasing, so active distribution structure is still becoming more and more complex, and the distributed characteristics are becoming more prominent. The new requirements for the autonomy, operability and control of system have been put forward [1]. At the same time, there are a large number of controllable distributed resources in active distribution network such as energy storage, flexible load and so on. Energy storage has the ability to transfer power and energy; therefore, it has become an effective way to solve the problem of renewable energy access. The introduction of energy storage system (ESS) in active distribution network can effectively compensate for the problem of power system fluctuation caused by distributed renewable energy [2] [3] [4]. Flexible load is one of the most promising interactive resources in active distribution network due to its initiative and control capability. Making the full use of flexible load can effectively improve the impact of DG on distribution network and improve the adoptability of active distribution network.

It is difficult to fully collect real time data of DG, energy storage and flexible load by the existing data acquisition system in active distribution network. At the same time, the output of Distributed Renewable Energy DG has obvious randomness and volatility. The coordinated response of energy storage and flexible load get decentralized, and the traditional centralized control method has slow communication. Therefore, because of the large amount of calculation, it is difficult to realize the coordinated control of decentralized and time-varying active distribution network system. The Multi-Agent System (MAS) relies on its good autonomy, adaptability and coordination, it can meet the real-time interaction of different stakeholders and adapt to the development trend of the active distribution network. With the distributed characteristics and autonomous decision, models of MAS have obvious advantages in solving the coordination and optimization of complex active power distribution networks [5]-[11]; the ref. [1] is based on increased activity and distribution of active distribution network, in order to increase the ductility of active distribution network, a multi agent control system for a new type of distribution network is proposed. Ref. [11] aims at the problem of low accuracy and scheduling risk of wind power forecasting, and puts forward the use of energy storage system (ESS) to improve the scale of wind power network, and evaluate its economic performance from two aspects of ESS operation benefit and income. In the ref. [12], a multi-agent system (MAS) control model with multi-class load response is constructed, which is based on the characteristics of high density of commercial users. The coordinated control of the commercial load is carried out with the goal of user's comfort; the distributed control of flexible load such as electric vehicle, air conditioner and water heater is discussed, which provides the basis for coordinated control of active distribution network. In Ref. [13] based on MAS, a distributed energy optimization model for active distribution networks, and control structure for regional 
autonomy and global coordination is proposed. The control strategy is studied from the global improved objective of the distributed energy and the active distribution network, the validity of the distributed control system to the active distribution network is proved, which improves the versatility and robustness of the system optimization model.

Therefore, based on the active distribution network security constraints, the market price guide, coordination of DG, energy storage and load and other benefits are important means to promote the utilization rate of Distributed Renewable Energy. Based on the hierarchical control structure of MAS, this paper proposes a distributed renewable energy coordination strategy based on price guidance and MAS regional agent target and active distribution network. It provides an effective method for the coordination of Distributed Renewable Energy consumption. Finally, the comprehensive performance of the proposed control strategy is evaluated by using the running index, and the validity of the MAS control model is verified by comparing the results of the traditional control method.

\section{MAS Hierarchical Control Framework Design}

\subsection{MAS Structure}

MAS is a kind of artificial intelligence computing method; its independent characteristics make it possible for electronic devices with common or conflicting goals to coexist in complex power systems. MAS based on JADE platform for Agent information interactive, distributed simulation based on JADE simulation platform has become an important research direction in recent years. JADE is to follow the FIPA specification, to achieve interoperability between Agents, while simplifying the process of MAS developing platform, Agent is the middleware to help Agent to achieve message encoding, message transmission, message resolution and message parsing work [4].

In this paper, the characteristics of MAS communication and decision ordnance are adopted. A multi-agent structure for coordinated control of DG, ESS and flexible loads in an active distribution network is proposed. The control structure is shown in Figure 1. The MAS hierarchical structure of multi-agent model is used to control the DG, energy storage and load, and the hierarchical multi-agent model is shown in Figure 2.

1) Lead Agent (DNO): Starting from the global target, coordinating regional agents, setting up the regional catalytic signal to achieve the overall goal maximization;

2) Region Agent (DG, ESS, FL): In response to the driving signal of the Agent, the regional autonomy is realized according to the regional target;

3) Controlled Agent: To achieve the DG, energy storage devices, flexible load control, response to the regional Agent instructions.

\subsection{MAS Coordination Process}

Traditional time series simulation belongs to a simple set of power flow calculation 


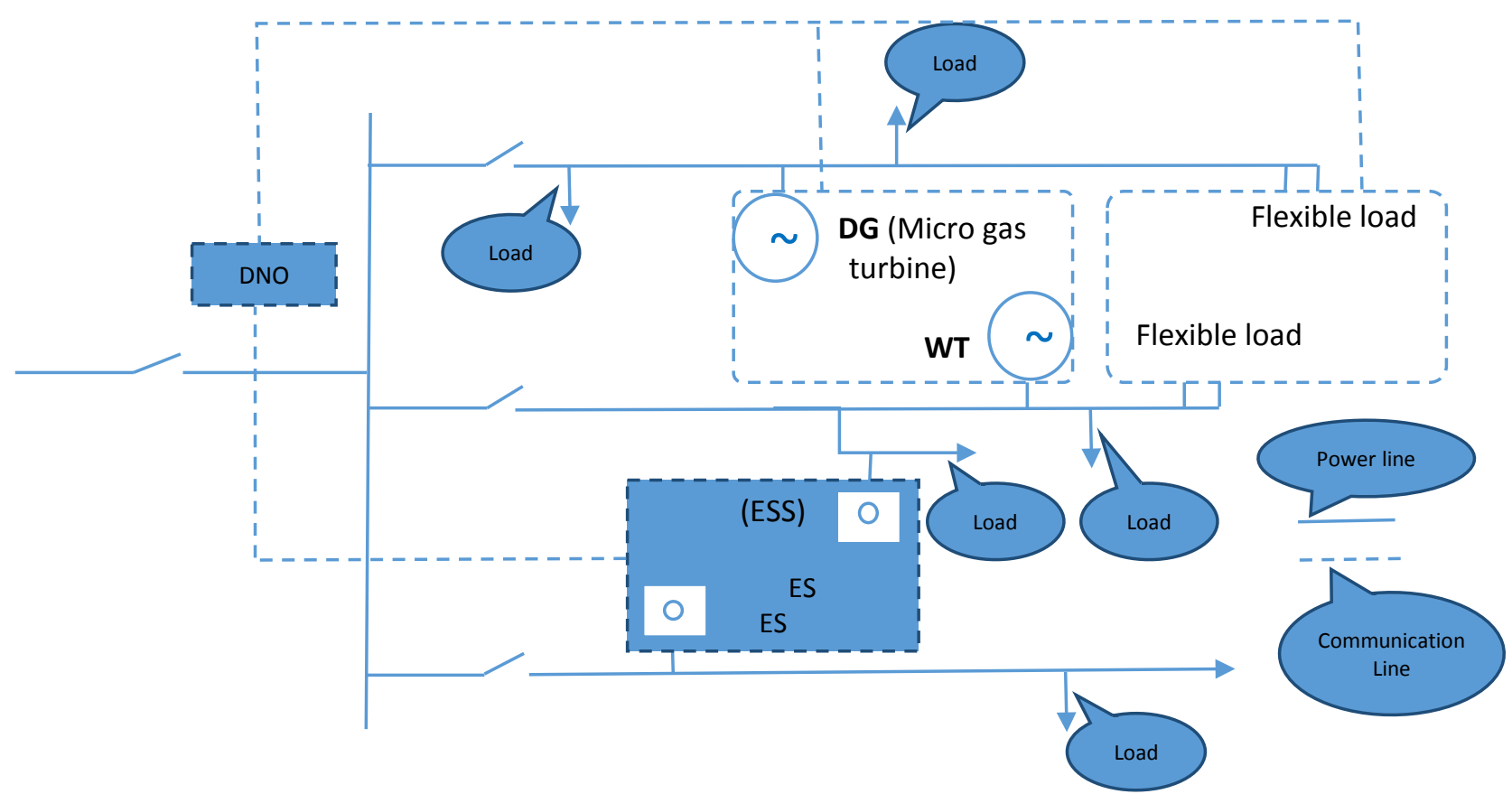

Figure 1. Multi-agent control structure in active distribution network.

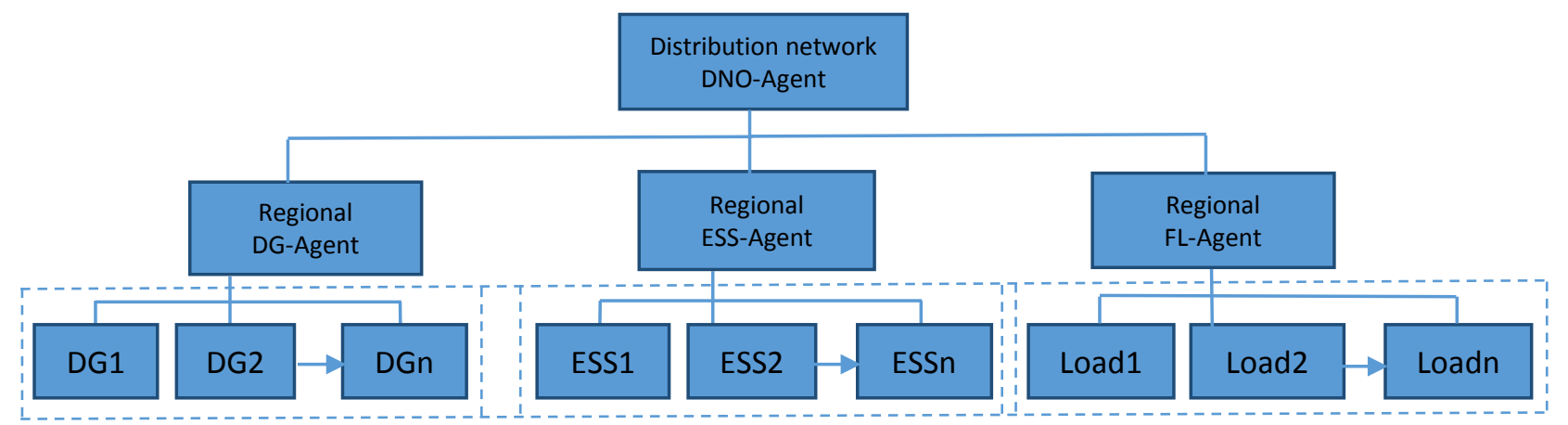

Figure 2. MAS hierarchical multi-agent model.

at each time point; therefore, it is difficult to use the traditional sequential simulation method to highlight the initiative of DG and energy storage in active distribution network, at the same time, it is difficult to reflect the interaction between DG, ESS and flexible load. Compared with the traditional control methods, the decentralized decision making of MAS increases the uncertainty of the active distribution system operation, at the same time, the operation state of the system is optimized by the mutual influence and adjustment skills between DG, energy storage and flexible load.

This paper focused on DG in active distribution network, the coordinated control of energy storage and load is put forward, and the scheme of intensive optimization and decentralized control for MAS is proposed; DNO-Agent is mainly responsible for coordinating the power balance of the whole distribution network system, the controlled equipment in the regional Agent coordination area is adapted to the DNO optimization objective. The coordination process is 
shown in Figure 3. DNO send electricity price and distribution network information to regional Agent, regional Agent achieves coordinated response and feedback response scheme according to regional autonomy goal, DNO collect regional response plan, check system security, and make plans to feedback area Agent; When the zone agent initiates an active request to DNO, DNO coordinates the other regional agents to develop the scheme that implements the response to the regional agent request in case of system security.

\section{Price Based Multi Agent Coordination Strategy}

\subsection{DNO-Agent Model}

DNO-Agent is responsible for monitoring and maintaining the power balance and system power flow safety of the whole distribution network system. DNO send electricity price and distribution network information to regional Agent; Based on ensuring of overall supply, demand balance, safe and stable operation of the active distribution network, DNO receive a request from the area Agent or provide a response plan. DNO aims at minimizing coordination costs.

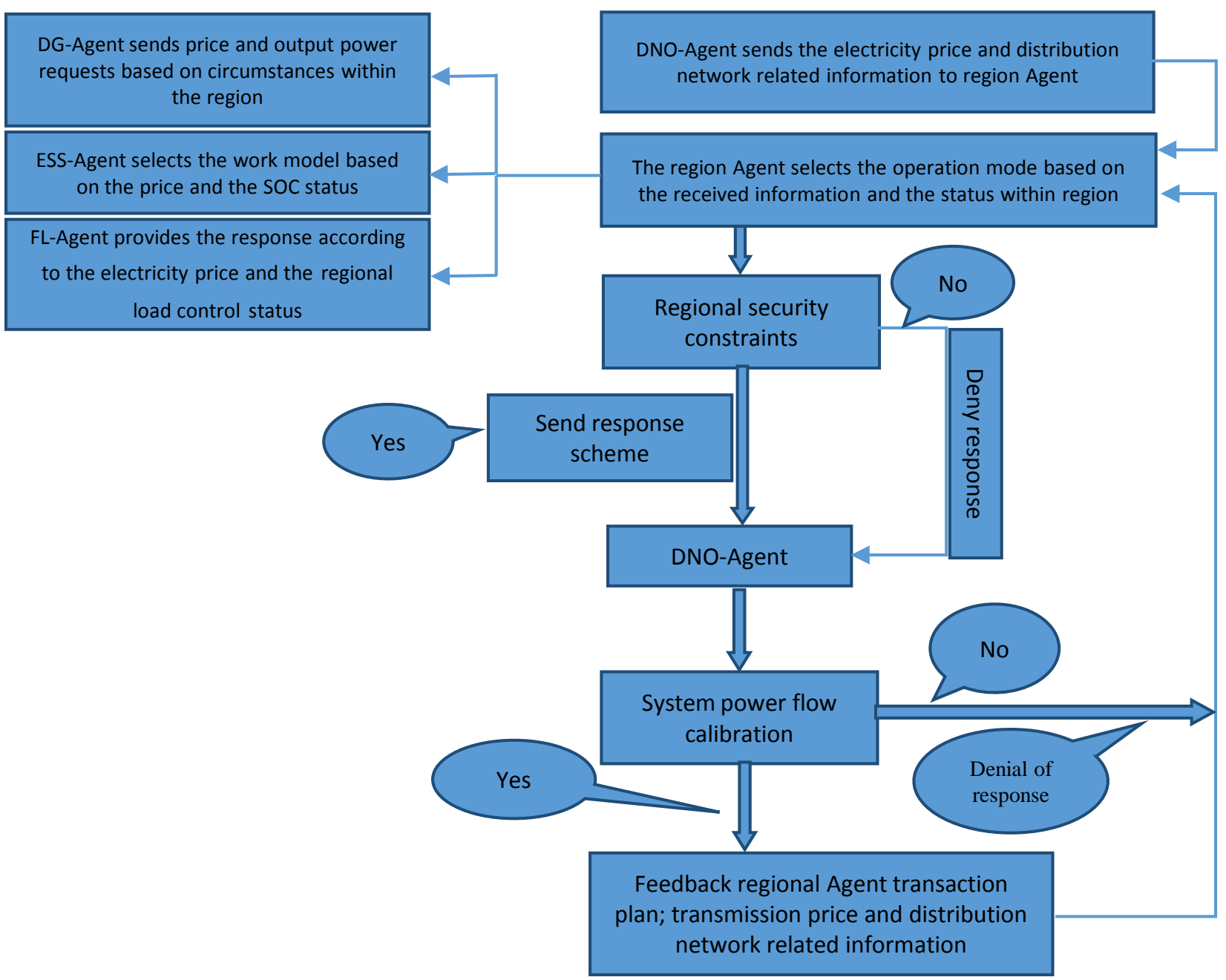

Figure 3. MAS coordinate control. 


$$
\begin{aligned}
& \min f=C_{t} P_{g r i d, t}+C_{t} P_{D G, t}+C_{t} P_{L, t}+C_{t} P_{E S, t} \\
& P_{g r i d, t}+P_{D G, t}=P_{L, t}+P_{E S, t}
\end{aligned}
$$

Type: $C_{t}$ is the price of $\mathrm{t}$ time; $P_{\text {grid }, t}$ is the power generation of the grid; $P_{D G, t}$ is the DG response; $P_{L, t}$ is the load response; $P_{E S, t}$ is the energy storage response, the charge is positive, and the discharge is negative.

System security constraints are as follows:

1) Active power and reactive power flow constraint

$$
\begin{aligned}
& P_{i}-V_{i} \sum_{j=1}^{N} V_{j}\left(G_{i j} \cos \theta_{i j}+B_{i j} \sin \theta_{i j}\right)=0 \\
& Q_{i}-V_{i} \sum_{j=1}^{N} V_{j}\left(G_{i j} \sin \theta_{i j}+B_{i j} \cos \theta_{i j}\right)=0
\end{aligned}
$$

Type: $P_{i}$ and $Q_{i}$ respectively for the $i$ node, active and reactive power; $N$ is the total number of nodes; $V_{i}$ is the $i$ node voltage; $V_{j}$ is the $j$ node voltage; $G_{i j}$ and $B_{i j}$ are $i j$ line conductance and electrical acceptance, respectively.

2) Node voltage constraints

$$
V_{i, \min } \leq V_{i} \leq V_{i, \max }
$$

Type: $V_{i, \min }, V_{i, \max }$ are node voltage lower and upper limit, respectively.

3) Branch power constraints

$$
\left|P_{t}\right| \leq P_{l, \max }
$$

Type: $P_{l, \max }$ is the upper limit of the transmission power of line $\mathrm{L}$.

\subsection{Regional Agent Model}

\subsubsection{DG-Agent}

DG-Agent achieves active response to DG energy management and active distribution network demand through coordinated control of distributed generation units in active distribution network. Distributed generation includes controllable power generation and uncontrollable power generation, in which uncontrolled power generation mainly refers to distributed renewable energy power generation. In the DG model, a controllable micro gas turbine and an uncontrollable fan generator are taken as examples. The wind turbine's output model simulates its output by using Monte Carlo and two-parameter Weibull, output power is

$$
P_{i, t}= \begin{cases}0 & V_{t}<V_{c}, V_{t}>V_{f} \\ R_{i}\left(\frac{V_{t}-V_{c}}{V_{s}-V_{c}}\right) & V_{c}<V_{t}<V_{s} \\ R_{i} & V_{s}<V_{t}<V_{f}\end{cases}
$$

where: $P_{i, t}$ is the output power of the first $i$ wind turbine at $t ; V_{t}$ the wind speed for the $t$ moment; $V_{c}$ is the cut in wind speed; $V_{f}$ is the cut out wind speed; $V_{s}$ is the rated wind speed; $R_{i}$ is the rated capacity of the first $i$ wind turbine. 
The micro turbine meets the power constraint:

$$
P_{M T, \min }<P_{M T, t}<P_{M T, \max }
$$

where: $P_{M T \text {,min }}$ is the minimum output power of the gas turbine; $P_{M T, t}$ are the actual output power of the gas turbine at $t$, and $P_{M T \text {, max }}$ Maximum output power for gas turbine.

DG-Agent collects and manages the output status information of the gas turbines and wind turbine in the area, and ensures the safe operation of all the equipment in the Agent. Among them; the wind turbine output is not controllable, Agent should be controlled to maximize its absorptive capacity as the goal; the output of the gas turbine is controllable, which is mainly used to meet the demand of the wind turbine and the stored energy when the output power is not enough. The DG-Agent coordinated control function is shown in Figure 4.

At the initial moment, DNO sends the grid price and system load demand to DG-Agent, DG-Agent collects the power generation status and output power of controlled DG, Feedback to DNO for its own responsiveness; DNO takes the system power balance as the constraint, coordinates the Agent response of each region, determines the purchasing power and feedback DG-Agent; DG-Agent accept the remaining amount of distribution power of wind machine for calculating purchasing power, it maximizes the implementation of renewable energy generation in the region, stimulate the active distribution network storage and load response efforts. According to the remaining power of wind turbines, DG-Agent adjust the price of electricity, Initiate the consumptive request to DNO; DNO coordinates other regional Agent to meet the demand response of DG-Agent, and feedback the coordination purchase plan to DG-Agent. The DG-Agent accepts the response scheme, and if the renewable energy is left within the security constraint, the consumer request is initiated again.

It is worth emphasizing that, The DG-Agent coordination strategy is based on the remaining power and market price of the wind turbine, which stimulates the

DG-Agent
1. Collect the power generation and
power generation status of DG in
this area
2. Receive the purchase plan, the
distribution of wind power machine
calculation of residual power; adjust
the price according to the amount
of residual, initiate consumptive
request
3. The clean energy surplus in the
area repeats step 2, and on the
contrary, the round ends with
coordination.

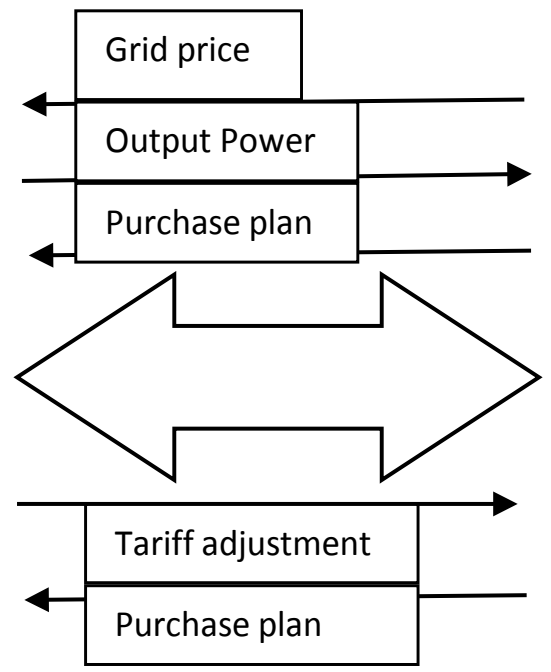

DNO-Agent
1. Transmission price and load
demand
2. Coordinate the Agent response;
determine the DG power
generation, and feedback
3. Receiving DG-Agent requests to
other regional consumptive, Agent
response, feedback and
coordination scheme

Figure 4. Coordination control of DG-Agent. 
energy storage and flexible load in the active distribution network to improve its consumption. When the wind turbine output surplus, the DG-Agent actively sends a power request to the DNO, the transmission of information including wind turbine power generation, the remaining adjusted price, the price adjustment is as follows:

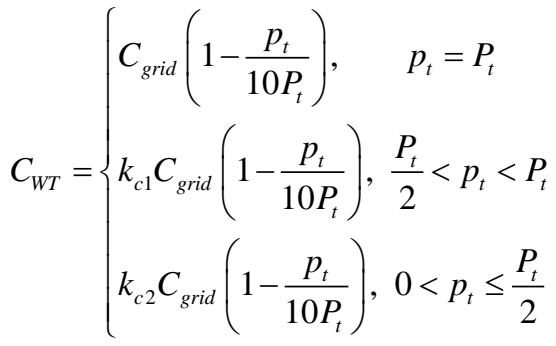

where: $C_{W T}$ is wind power price; $C_{g r i d}$ is power grid tariff; $k_{c 1}$ and $k_{c 2}$ are price adjustment factors; $P_{t}$ is total power wind turbine for t of time; $p_{t}$ is residual or remaining power of wind turbine for time $t$.

\subsubsection{ESS-Agent}

ESS-Agent collects and manages the status information of the energy storage devices in the region, and coordinates the demand of the active distribution network on the basis of ensuring the safe operation of the energy storage, maximize the consumption of wind turbine power. Energy management is realized in the ESS-Agent to maximize the energy storage revenue in the area. Energy storage devices are generally limited by the capacity and storage charge status, and to extend life of the energy storage device in the system, overcharge and over discharge is not allowed. The state function is

$$
S O C_{i, t+1}=S O C_{i, t}+\eta_{c h} P_{c h, i, t} \Delta t-\eta_{d i s} P_{d i s, i, t} \Delta t
$$

Restrictions:

$$
\left\{\begin{array}{l}
0 \leq P_{c h, i, t} \leq P_{c h, i, \max } \\
0 \leq P_{d i s, i, t} \leq P_{d i s, i, \max } \\
S O C_{i, \text { min }} \leq S O C_{i, t} \leq S O C_{i, \text { max }}
\end{array}\right.
$$

where: $S O C_{i, t}$ for $i$ storage device at $t$ state of charge (SOC); $P_{c h, i, t}$ and $P_{d i s, i, t}$ respectively in $t$ device time charging and discharging power of the $i$ storage; $\eta_{c h}$ and $\eta_{d i s}$ the charge and discharge efficiency; $P_{c h, i, \max }$ and $P_{d i s, i, \max }$ the charge and discharge power storage device; $\Delta t$ for the duration of the current state; $S O C_{i, \min }$ and $S O C_{i, \max }$ are the minimum and maximum allowable energy storage devices, Maximum charge state.

At the initial moment, DNO sends the power grid price to the ESS-Agent. Within the scope of security constraints, ESS-Agent determines the energy storage, discharge status and capacity of the stored energy according to the state of the energy storage device in the region and the price of the power grid, and feedback its response to the DNO; when DG-Agent initiate the launch request to DNO, DNO sends the wind power adjustment price and the remaining power 
generation to the ESS-Agent; ESS-Agent determines the storage state and power of stored energy according to the state of energy storage and the price of wind energy.

In the ESS-Agent regional control strategy, the charge and discharge behavior of the energy storage device is guided by the state of storage energy and the price of electricity, the aim of maximizing consumption of wind power and increasing its own income. The working state of the energy storage in the period is determined by considering the difference of the electricity price in different periods and the SOC state of the energy storage device in the current period, and the selection of the working strategy is shown in Table 1. ESS-Agent price based on DNO and information of active distribution network, combined with the state of energy storage to choose their own working state, at the peak load, the coordinated energy storage discharge can not only alleviate the system power supply pressure, but also maximize the energy storage benefits; When the load is low, the energy storage and charging is coordinated, which realizes the consumption of renewable energy, reduces the peak and valley load difference of the active distribution system, and reduces the charging cost. This strategy ensures safe operation of stored energy in the region, and based on income, improve the ability of the consumptive Distributed Renewable energy.

\subsubsection{FL (Flexible Load) Agent}

The behavior of flexible load is influenced by user's preferences and environmental factors. It has strong controllability and is easily guided by electricity price and other environmental factors; through the coordination of flexible load control can realize the active power distribution system peak, and contribute to the consumptive renewable energy output.

FL-Agent collects and manages the status information of the flexible load in the area, in order to ensure the comfort of the users; the system controls the flexible load to respond to the DNO demand. At the initial time, the DNO sends

Table 1. Control of energy storage.

\begin{tabular}{|c|c|c|c|}
\hline Time period & Electricity price & Energy storage SOC & Energy storage status \\
\hline Valley/low time & Grid price & $0.2 \leq \mathrm{SOC}<0.5$ & Normal charging \\
\hline$=$ & Grid price & $0.5 \leq \mathrm{SOC}<0.85$ & Low power charging \\
\hline \multirow{2}{*}{$=$} & Wind energy price & $0.2<\mathrm{SOC}<0.5$ & Limit charge \\
\hline & adjustment & $0.5 \leq \mathrm{SOC}<0.85$ & Normal charging \\
\hline Usual time & Grid price & I & Stop working \\
\hline$=$ & $\begin{array}{c}\text { Wind energy price } \\
\text { adjustment }\end{array}$ & $0.2 \leq \mathrm{SOC}<0.5$ & Normal charging \\
\hline \multirow{2}{*}{ Peak time } & \multirow{2}{*}{ Grid price } & $0.5 \leq \mathrm{SOC}<0.85$ & Limit discharge \\
\hline & & $0.2 \leq \mathrm{SOC}<0.5$ & Normal discharge \\
\hline$=$ & $\begin{array}{l}\text { Wind energy price } \\
\text { adjustment }\end{array}$ & I & Stop working \\
\hline
\end{tabular}


the power grid price to the FL-Agent, and the FL-Agent sends the response to the DNO according to the load demand in the area; When DG-Agent launched the consumptive requests to DNO, DNO send the wind power tariff adjustment and surplus generation to FL-Agent; FL-Agent responds the load response according to the state of the flexible loads and the safety constraints in the area. The response model of the flexible load in the model is

$$
\begin{gathered}
P_{L, t}=\varepsilon \frac{C_{t}-C}{C} P_{\text {Load }, t} \\
C=\frac{\sum_{T} P_{\text {Load }, t} C_{t}}{\sum_{T} P_{\text {Load }, t}}
\end{gathered}
$$

where: $P_{L, t}$ are the response quantities of flexible loads at $t$ moments; $P_{\text {Load, } t}$ is the total amount of flexible load in FL-Agent at time $t, \varepsilon$ is the overall price elasticity coefficient of the flexible load; $C$ for the comprehensive price; $T$ is the whole work cycle; $C_{t}$ is the current grid price.

\subsection{Price Based Interregional Coordination}

When the active energy distribution network requests cannot be achieved within the DG Agent area distributed renewable energy consumption, the DG-Agent interacts with the DNO-Agent through the protocol mechanism to achieve the interactive protocol to realize the consumption of regional distributed renewable energy. There are two difficulties in the storage of renewable energy:

1) Hard mode: regional renewable energy consumptive is difficult, active distribution network energy coordination of renewable energy power surplus for $p_{t} \geq 0.5 P_{t}$. In order to achieve the distributed renewable energy consumption, reduce the abandoned wind, renewable energy should seek new response to the consumptive surplus. In the difficult mode, the amount of renewable energy power generation is large, Negative energy storage in the system, Flexible load again respond to the pressure of large consumption, DG-Agent significantly reduce the electricity price, The price adjustment Formula (7), hope to lower prices to stimulate and improve the system response efforts to promote renewable energy consumption.

2) Easy mode: regional renewable energy consumptive is easy, active distribution network energy coordination of renewable energy power surplus for

$p_{t}<0.5 P_{t}$. In an easy way, renewable energy generates less power, the pressure of load and energy storage is less, and DG-Agent reduces the price of electricity properly. The price adjustment is shown in type (7), in the promotion of renewable energy consumption while ensuring the economic benefits of DG agents. When renewables generate electricity, the DG-Agent adjusts the electricity price, DNO-Agent requests power consumption; DNO-Agent sends a request for renewable energy to other regional agents; ESS-Agent determines the working state of the energy storage according to the electricity price and the charged state of the stored energy in the region; FL-Agent determines the response of the re- 
gional load according to the change of electricity price; DNO receives the energy storage charge and the load response feedback, Check system flow, If the security returns DNO-Agent accommodation scheme, On the contrary, the DG-Agent refused to accept the request, requiring the wind.

\section{Operational Level Evaluation}

Operational level evaluation includes performance evaluation of active distribution network in terms of operating voltage level, system load and component economy. The comparison and analysis of different control methods not only need to compare with the index, but also analyze the whole performance. However, the dimension and nature of the evaluation indexes of active distribution network are different, and not all the indexes can be analyzed directly. Fuzzy Hierarchy Process, FAHP to achieve the complex multi-factor co-scale analysis, the subjective influence of the decision maker is even lower.

\subsection{Fuzzy Comprehensive Analysis}

FAHP is a combination of quantitative and qualitative analysis, and is suitable for multi-objective decision-making, It can analyze the non-sequential relations among multiple objectives, and avoid the bias caused by the subjective limitations of the analytic hierarchy process; it establishes the fuzzy consistency judgment matrix through the comparison of the influencing factors, the non-correlation qualitative relation between the indexes of distribution network is changed into quantitative weight relation, and reduce the influence of subjective definition weight value on the analysis result. In this paper, a fuzzy consistent judgment matrix is established by comparing the 22 of the influencing factors [13], Quantitative conversion values for qualitative relationships between indicators are shown in Table 2.

The weight value of the index is calculated by using relation scale between indexes

$$
\omega_{i}=\frac{\coprod_{j=1}^{M} r_{i j}}{\sum_{i=1}^{M} \prod_{j=1}^{M} r_{i j}^{\frac{1}{M}}}
$$

Table 2. Relationships between elements.

\begin{tabular}{crl}
\hline Scaling $\boldsymbol{r}$ & Definition & \multicolumn{1}{c}{ Description (compared the two elements) } \\
\hline 0.5 & Equally important & Equally important \\
0.6 & Slightly important & An element is a little more important than another \\
0.7 & Obviously important & One element is more important than another \\
0.8 & very important & One element is very important than another element \\
0.9 & Extremely important & One element is more important than the other \\
$0.1,0.2,0.3,0.4$ & Inverse comparison & If the element $a_{i}, a_{j}$ is compared to $r_{i j}$, \\
& & Then the element $a_{j}, a_{i}$ is compared to $r_{j i}=1-r_{i j}$ \\
\hline
\end{tabular}


where: $\omega_{i}$ is the index weight value; $M$ is the total number of evaluation indexes; $r_{i j}$ is the scaling relation between two indexes.

Convert all index results to non-dimensional and impactful values. The comprehensive evaluation score of the operation strategy is obtained by using the weight coefficient and the effective value of the index, the formula is

$$
S=100 \sum_{i=1}^{M} \omega_{i} X_{i}
$$

where: $V_{V a r}$ voltage variance; $Z_{i j}$ line impedance; $Q_{i}$ line $i j$ terminal reactive load; $V_{i}$ line $i j$ first terminal voltage; $X_{i j}$ Line $i j$ Reactance; $n$ Number of load nodes; $D(i)$ System node load value; $b(i)$ Stored energy output power; $K$ Energy consumptive capacity; $m$ Total energy storage device; $t_{L}$ Total load trough; $\Delta P_{i, D G, t}$ Wind energy charge; $B_{1}$ Energy arbitrage gains; $C_{c h, t}, C_{d i s, t}$ Charge (discharge price); $P_{\text {dis, },}, P_{c h, t}$ Charge (discharge power).

\subsubsection{Voltage Operating Level}

Voltage operating level includes system voltage deviation, Reactive stability. It is the main index to measure the quality of the power supply of the multi type energy in the active distribution network. The guarantee voltage excursion is the basic task of active power distribution system operation adjustment [14].

The uncertainty of clean energy output and load fluctuation exacerbates the fluctuation of grid voltage largely [15]. In actual operation, adjustment of operating mode, DG and load timing fluctuations will lead to node voltage offset, the voltage deviation has a negative effect on the equipment in the system. In addition, the reactive load will also affect the stability of the system voltage, and its change will lead to sudden abrupt change in voltage.

\subsubsection{Operating Peak Valley Difference}

The introduction of renewable energy into the active distribution network is a problem that the power system has been trying to solve. The effective control of energy storage and load in active distribution network can reduce the negative

Table 3. Evaluation indicators.

\begin{tabular}{|c|c|c|}
\hline Index & Formula & Significance \\
\hline $\begin{array}{l}\text { Voltage } \\
\text { deviation }\end{array}$ & $V_{\text {Var }}=\frac{1}{N} \sum_{i=1}^{N}\left(V_{i}-\frac{1}{N} \sum_{j=1}^{N} V_{i}\right) 2$ & $\begin{array}{l}\text { Reflect the degree of } \\
\text { fluctuation of node voltage }\end{array}$ \\
\hline $\begin{array}{l}\text { Reactive power } \\
\text { stability }\end{array}$ & $V S I=\frac{4 Z_{i j}^{2} Q_{j}}{V_{i} X_{i j}}$ & $\begin{array}{l}\text { Measuring system } \\
\text { reactive stability }\end{array}$ \\
\hline Load transfer & $f=\frac{1}{n} \sum_{i=1}^{n}\left\{[D(i)+b(i)]-\frac{1}{n} \sum_{j=1}^{N}[D(j)+b(j)]\right\} 2$ & $\begin{array}{l}\text { Measure the smoothness } \\
\text { of a load curve }\end{array}$ \\
\hline $\begin{array}{l}\text { Energy storage } \\
\text { benefits }\end{array}$ & $K=\frac{\sum_{t_{L}} \sum_{i=1}^{m} \Delta P_{i, D G, t}}{\sum_{i=1}^{m} R_{i}}$ & $\begin{array}{l}\text { When the low load can } \\
\text { reflect the absorptive } \\
\text { capacity of the energy } \\
\text { storage device }\end{array}$ \\
\hline $\begin{array}{l}\text { Wind energy } \\
\text { consumption }\end{array}$ & $B_{1}=\sum_{T} C_{d i s, t} P_{d i s, t} t-\sum_{T} C_{c h, t} P_{c h, t} t$ & $\begin{array}{l}\text { Evaluate the economy of } \\
\text { the energy storage system }\end{array}$ \\
\hline
\end{tabular}


impact of intermittent energy and promote the permeability of distributed renewable energy in power grid. On the other hand, the system can effectively adjust the peak and valley load difference, and alleviate the power supply pressure at the peak load of the distribution network.

Clamping peak filling is an important task of active distribution network optimization, which is an important index to measure the stability and reliability of distribution network. Peak daytime load, The energy storage discharge can effectively alleviate the load generation pressure on the generator and avoid the full load operation of the generator while reducing the system load shedding rate; When the night load is low, the randomness of wind power generation and the capacity of the system load are reduced. When the night load is low, the randomness of wind power generation and the reduced capacity of the system load are caused by the wind blows. The energy storage charge plays the role of "load", which consumes excess wind energy and improves the utilization rate of wind energy, and for the daytime load peak energy storage discharge energy savings. For the power system, reducing the peak load difference can effectively delay the upgrading of power equipment capacity, improve equipment utilization, and reduce system cost. Furthermore, evaluation indexes and its significances are shown in Table 3.

\subsubsection{Energy Storage Arbitrage}

Because of the randomness and volatility of renewable energy sources, the presence of ESS is essential; the introduction of energy storage in active distribution network can increase the utilization rate of renewable energy and smooth load curve, and is an effective means to adjust the voltage and frequency of the system [16]. The high cost limits the large-scale application of energy storage in distribution network, and the maximization of profit with limited capacity is the goal of energy management optimization of active distribution network. It is also an important index to evaluate the energy storage economy systematically.

\section{Case Study}

\subsection{Simulation Condition}

In this paper, an active distribution network system with IEEE33 nodes is used. The validity and effectiveness of multi-agent operation control model and strategy for active power distribution network are analyzed and verified, the structure of the active distribution network is shown in Figure 5, and the installation information of DG and energy storage is shown in Table 4. In order to prolong the service life of the energy storage device, set $S O C_{i, \min }=0.2, S O C_{i, \max }=0.85$, The initial state of stored energy is $S O C_{i, 0}=0.5$.

\subsection{Simulation of Coordinated Control Based on MAS}

In this paper, the peak price is adopted as the example, and the electricity price is shown in Table 5. In the model, DG, energy storage and load are based on the price to guide the formulation of Agent coordinated control strategy. 


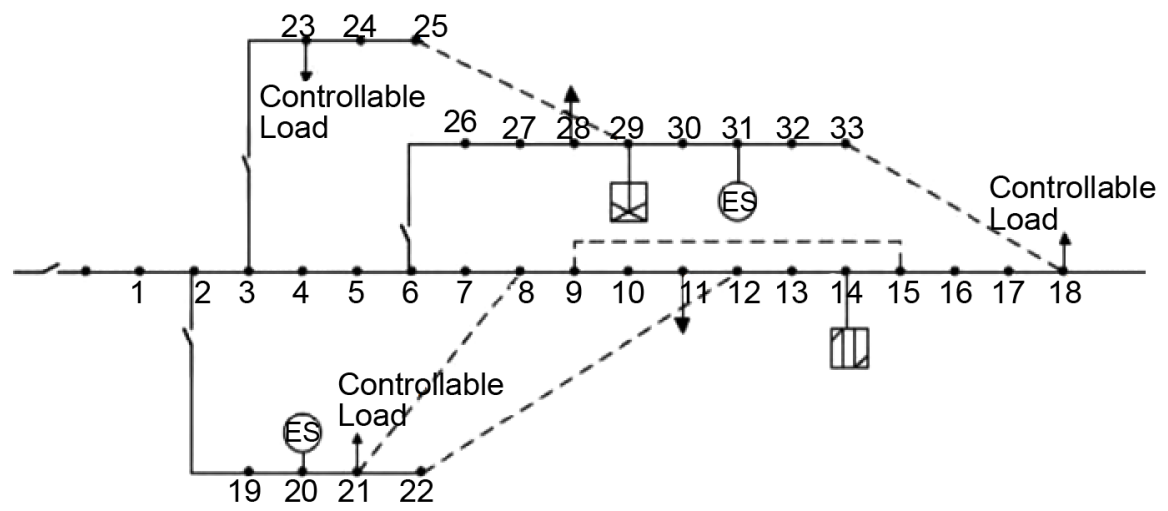

Figure 5. Active distribution network (AND) structure.

Table 4. The installation information of DG and ES.

\begin{tabular}{ccc}
\hline Installation node & Installation type & Capacity/MW \\
\hline 14 & Micro Gas Turbine & 0.3 \\
29 & WT & 0.5 \\
20 & ES & 0.1 \\
31 & ES & 0.1 \\
\hline
\end{tabular}

Table 5. Electricity price of grid, kWh.

\begin{tabular}{ccc}
\hline Valley & Peacetime & Peak time \\
\hline $23: 00 \sim 05: 00$ & $06: 00 \sim 07: 00$ & $08: 00 \sim 14: 00$ \\
0.415 & $15: 00 \sim 19: 00$ & $20: 00 \sim 22: 00$ \\
& 0.625 & 0.8745 \\
\hline
\end{tabular}

The simulation process of hierarchical control model for active distribution network is as follows: At the beginning of each period, the DNO-Agent initiates the request behavior in the MAS system and sends the price and the distribution network demand to the JADE service platform; DG-Agent collects the available electricity of the wind generator in the area, and makes the bidding strategy according to the electricity price and the electricity quantity, and feedback to service platform, and after the end of the single round bidding sale, if the remaining power, according to the remaining amount of electricity to modify the quotation, re apply to the DNO, to develop a new round of plans; ESS-Agent collects the status information of all the energy storage in the region, and develops the bidding scheme according to the current price and the energy storage state; FL Agent responds to the market price and the total controllable load at that time. The specific coordination process is shown in Figure 6.

It is worth emphasizing that, despite the adjusted schedule, DNO-Agent calculation is generated, and the system power flow security check is also generated by DNO, However, this is based on the operation data, data and coordination scheme provided by Agent in different regions. Compared with the centralized 


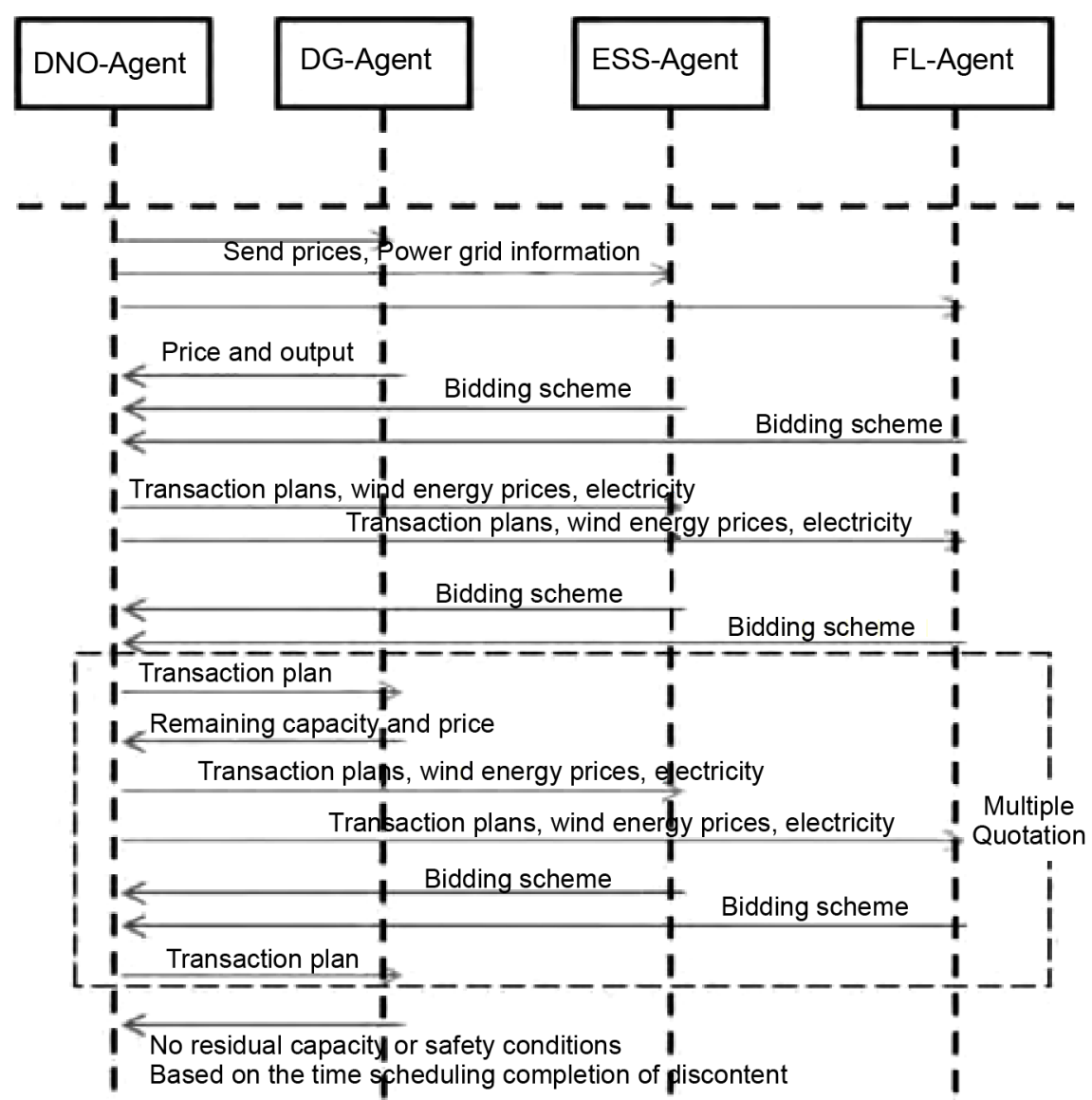

Figure 6. Realization of coordinated control for active distribution network.

control, the centralized to decentralized model adopted in this paper can disperse the complex optimization process, at the same time, the processing speed of the system is faster and the operation difficulty is reduced.

\subsection{Traditional Operation Model}

In traditional operation model, the installation node, capacity and electrovalence of DG and energy storage, the wind turbine output model and the initial energy storage state are consistent with the model proposed in this paper. Each time period, the wind turbine and the micro gas turbine deliver the power generation to the distribution network, then the distribution network must force its output, DG output and consumption without price adjustment; the energy storage working state is related to the current storage energy, SOC and the power grid price, but stored energy can only be operated at one time. The output of distributed renewable energy sources does not have active regulation capability; in addition, the system does not have a flexible load under active management.

\subsection{Results and Analysis}

The load peak and valley difference of the active distribution network system leads to the increase of the investment cost and the decrease of the operation 
safety and stability. As can be seen from Figure 7, the peak load difference of the distribution system is about 9.57 MW in the traditional model, the system load peak and valley difference of the MAS control model mentioned in this paper is only 7.4 MW, compared to the traditional model of the peak load is reduced by $22 \%$, therefore, this paper proposed a distributed control strategy is effective in regulating the system load than the traditional control strategy.

Figure 8 is the energy storage based on the ESS-Agent control model charging and discharging behavior and energy price, as can be seen from the diagram, the selling price of the wind power is different from the price of the power grid

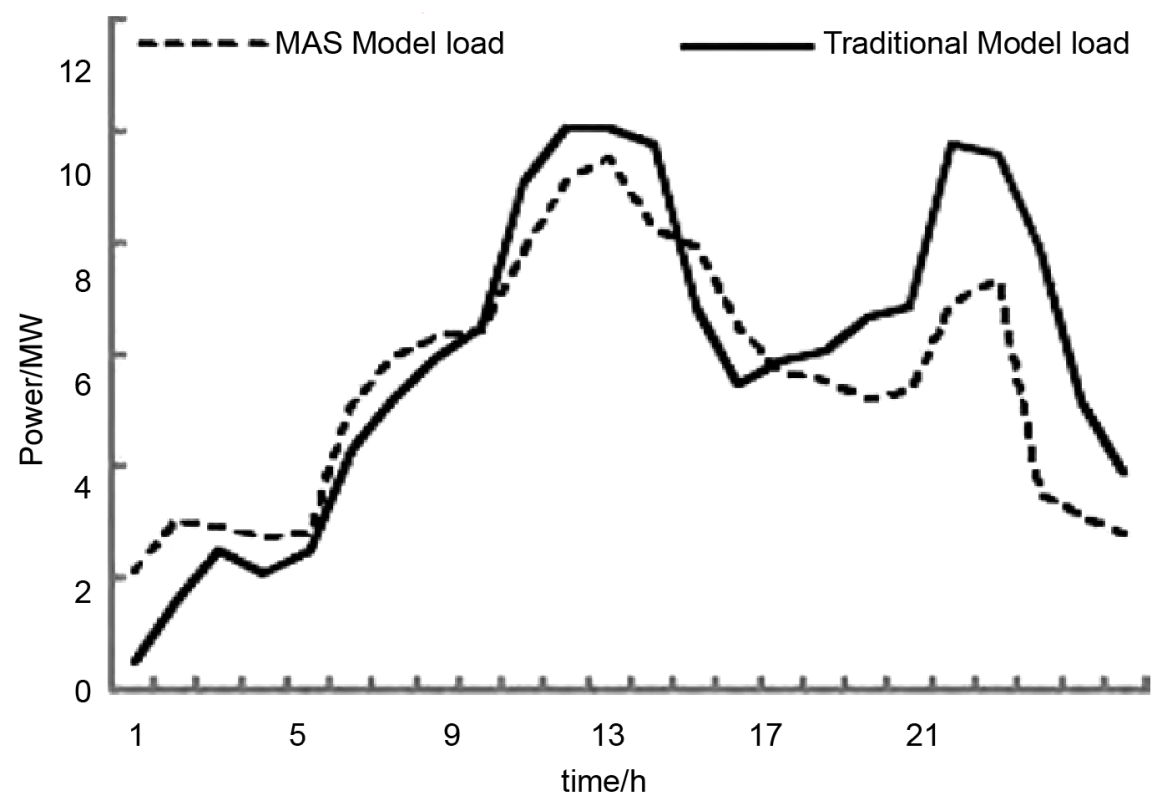

Figure 7. Systematic load comparison of different control measures.

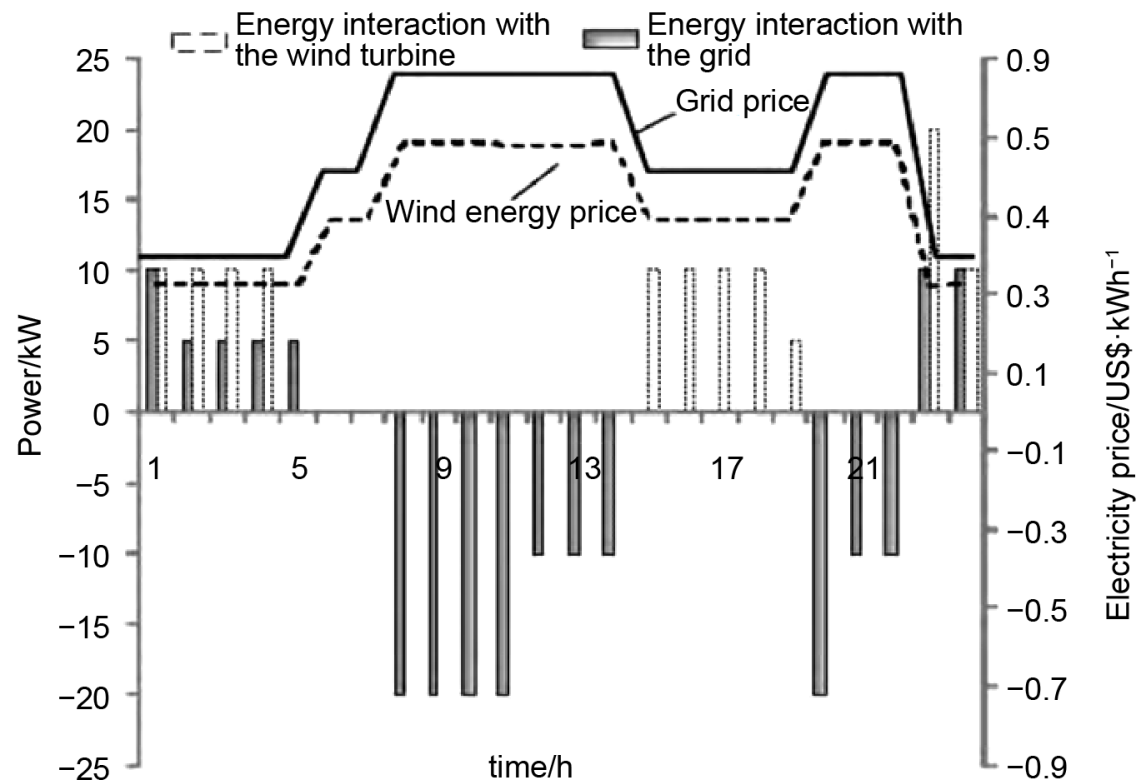

Figure 8. Energy storage coordination process in MAS. 
at different times, When the power grid is at peak time, the wind turbine output is large, the energy storage discharge is flexible and the load response is flexible. In order to improve the amount of wind energy consumption, wind energy prices and grid price difference as high as $0.03 \mathrm{US} \$ / \mathrm{kWh}$; When the power grid is in the valley, the electricity price, At the same time, consumption of energy storage and load, the price difference between wind energy and power grid is only $0.01 \mathrm{US} \$ \mathrm{kWh}$; Compared to the storage and discharge state of single energy storage centralized control, shown in Figure 9, MAS model of energy storage in power grid and electricity by wind power is composed of two parts, it directly reflects the storage of renewable energy consumption. In addition, the control strategy adopted in this paper increases the uncertainty of energy storage behavior, and fully explores the active decision-making ability of ESS-Agent to energy storage behavior, it makes the price guided ESS-Agent decision model more economical.

In this paper, the relationship between the element scale and the index weight is shown in Table 6. The operation performance of the distribution network under two control modes is evaluated by using the evaluation index, and the results are shown in Table 7. As can be seen from Table 6, the voltage operation level accounts for the largest value of the weight, and the economic value of the weight is the smallest, which conforms to the basic requirements of the safe operation of the power system.

Table 7, the load transfer index based on MAS coordination is only $15.2 \%$ of the traditional control model. It shows that the distributed control method has a significant effect on stabilizing the load fluctuation, and can effectively smooth the load curve; In addition, the voltage deviation index is $41.7 \%$ of the traditional control model, and the proposed method is more effective in controlling the

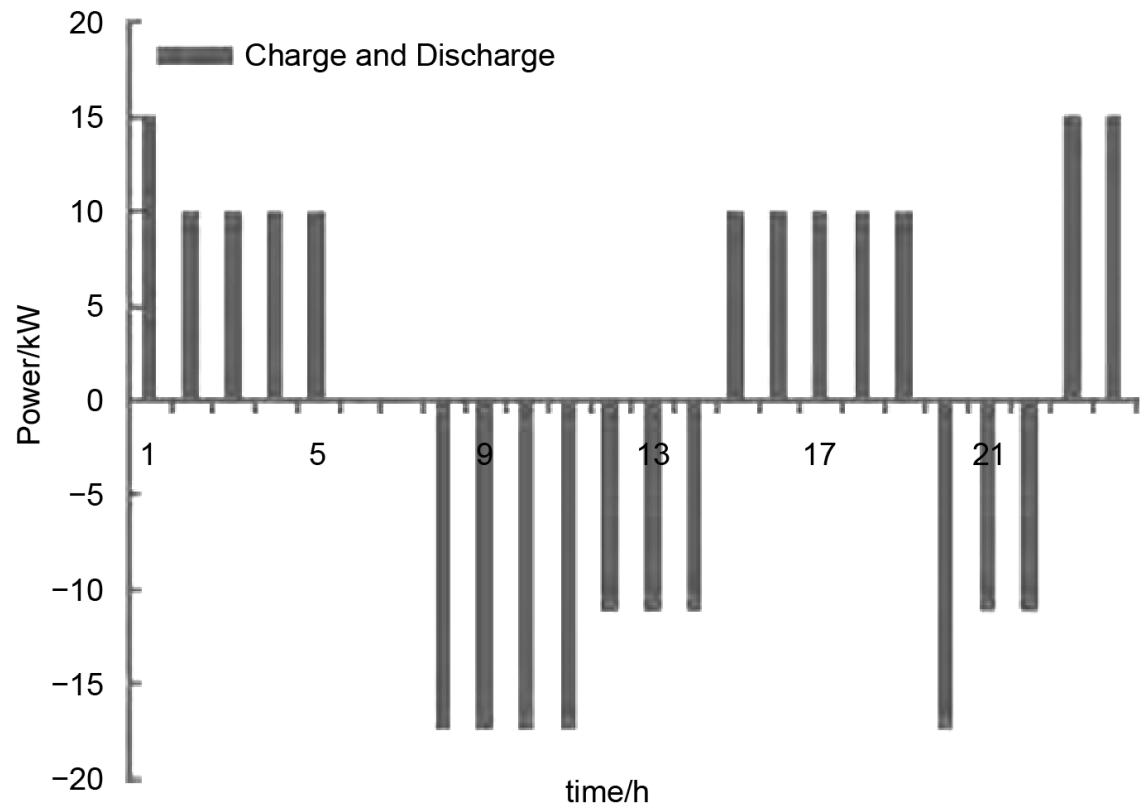

Figure 9. Energy storage coordination process of traditional model. 
Table 6. Relationship between evaluation indexes and index weight.

\begin{tabular}{ccc}
\hline Voltage level & Peak load difference & Economy of energy storage \\
\hline Voltage 0.5 & 0.7 & 0.9 \\
Load 0.3 & 0.5 & 0.6 \\
Economic efficiency 0.1 & 0.4 & 0.5 \\
Weight value 0.4016 & 0.322 & 0.2763 \\
\hline
\end{tabular}

Table 7. Index analysis of different control modes.

\begin{tabular}{ccc}
\hline Quantitative index & MAS model & Traditional model \\
\hline Voltage deviation (max) & 0.0247 & 0.0592 \\
Reactive power stability (max) & 0.73 & 0.7743 \\
Load transfer (max) & 0.0232 & 0.153 \\
Wind energy consumption & 0.35 & $/$ \\
Energy storage arbitrage/US\$ & 3755 & 3293 \\
Composite score (100 Point system) & 87.89 & 77.55 \\
\hline
\end{tabular}

node voltage of active distribution network, and can effectively adjust the distributed DG, The adverse effects of load fluctuation on voltage improve the stability and power quality of active distribution network; From the stability index, the index values of all the nodes in the two models are stable at about 0.7 , However, this strategy cannot improve the reactive power stability in the system.

The introduction of energy storage has promoted the rapid development of multi type power generation in active distribution network, and the core of energy storage coordination control is to explore the storage capacity and improve the storage economy. The comparison of energy storage arbitrage values from Figure 8 and two control methods is based on the ESS-Agent energy storage control strategy, the energy storage system's annual revenue was $14 \%$ higher than the traditional model, we can see that the economy of the MAS model of energy storage in higher; The model presented in this paper can clearly monitor the actual working state and energy sources of energy storage devices, and reflect the promotion of energy storage to improve the permeability of Distributed Renewable Energy sources.

In addition, the use of Fuzzy Analytical Hierarchy Process (FAHP) to calculate the comprehensive score of two models, 10.34 points higher than the traditional MAS model, it is proved that the proposed control strategy of MAS based on active distribution network operation control is better than the traditional model control strategy.

\section{Conclusion}

In this paper, a price guidance coordination control strategy based on MAS is proposed. Considering the output characteristics of DG and the response cha- 
racteristics of energy storage and load, a regional multi-agent model is established, and the energy management optimization of active distribution network is realized under the market mechanism; Furthermore, the performance of active distribution network is analyzed by using index. Compared to the traditional centralized control method, this model uses multi-agent information exchange characteristics, and increases the active distribution network operation uncertainty, making the simulation run process closer to the actual state; at the same time, the price factor is used to guide the coordination of the energy in the active distribution network, which highlights the active response capability of the energy storage and load, and increases the cooperation between the components. In addition, the evaluation indexes quantify the difference between the distributed control method and the traditional centralized control method, and it provides a train of thought for the subsequent research to choose the merits of different planning schemes.

\section{References}

[1] Prostejovsky, A., Merdan, M. and Schitter, G. (2013) Demonstration of a Multi-Agent-Based Control System for Active Electric Power Distribution Grids. IEEE International Workshop on Intelligent Energy Systems, 14 November 2013.

[2] Shi, T., Zhang, B., Chao, Q., et al. (2016) Economic Storage Ratio and Optimal Control of Hybrid Energy Capacity Combining Stabilized Wind Power Fluctuations with Compensated Predictive errors. Power System Technology, 40, 477-483.

[3] Atwa, Y.M. and El-Saadany, E.F. (2000) Optimal Allocation of ESS in Distribution Systems with a High Penetration of Wind Energy. IEEE Transaction on Power System, 25, 1815-1822. https://doi.org/10.1109/TPWRS.2010.2045663

[4] Yan, G., Liu, J., Cui, Y., et al. (2013) Economic Evaluation of Improving the Wind Power Scheduling Scale by Energy Storage System. Proceedings of the CSEE, 33, 45-52.

[5] Logenthiran, T., Srinivasan, D., Khambadkone, A.M., et al. (2012) Multi Agent System for Real-Time Operation of Amicrogrid in Real-Time Digital Simulator. IEEE Trans Smart Grid, 3, 925-933. https://doi.org/10.1109/TSG.2012.2189028

[6] Li, J., Song, X. and Meng, X. (2015) Hierarchical Control Model of Smart Distribution Network Based on Self-Organizing Multi-Agent System. In: International Conference on Renewable Power Generation, North China Electric Power University Press, Beijing, 1-6.

[7] Bellifemine, F., Poggi, A. and Rimassa, G. (1999) JADE-A FIPA-Compliant Agent Framework. C Selt Internal, 31, 103-128.

[8] Dimeas, A.L. and Hatziargyriou, N.D. (2005) Operation of a Multi Agent System for Mi Crogrid Control. IEEE Transactions on Power Systems, 20, 1447-1455. https://doi.org/10.1109/TPWRS.2005.852060

[9] Jiang, R., Qiu, X. and Li, D. (2014) Multi-Agent System Based Dynamic Game Model of Smart Distribution Network Containing Multi-Microgrid. Power System Technology, 38, 3321-3327.

[10] Hao, Y., Wu, Z., Dou, X., et al. (2012) Application of Multi-Agent Systems to the DC Microgrid Stability Control. Proceedings of the CSEE, 32, 27-35.

[11] Yuan, T., Chen, J., Liu, P., et al. (2014) Strategy of Improving Large-Scale Wind 
Farm Output Fluctuation Based on Energy Storage System. Power System Protection and Control, 42, 47-53.

[12] Yu, N., Yu, L. and Li, G. (2015) Controllable Load Management Strategy for Commercial Users Based on Multi-Agent in Smart Grid Environment. Automation of Electric Power Systems, 39, 89-95.

[13] Pu, T., Liu, K., Li, Y., et al. (2015) Multi-Agent System Based Simulation Verification for Autonomy-Cooperative Optimization Control on Active Distribution Network. Proceedings of the CSEE, 35, 1864-1874.

[14] Lan, J., Xu, Y., Huo, L., et al. (2006) Research on the Priorities of Fuzzy Analytical Hierarchy Process. Systems Engineering-Theory \& Practice, No. 9, 107-112.

[15] Chen, F., Liu, D. and Chen, Y. (2015) Hierarchically Distributed Voltage Control Strategy for Active Distribution Network. Automation of Electric Power Systems, 39, 61-67.

[16] Wang, C., Sun, W., Yi, T., et al. (2013) Review on Energy Storage Application Planning and Benefit Evaluation Methods in Smart Grid. Proceedings of the CSEE, 33, 33-41. 